Teil 5

Maßnahmen zur Korrektur und Eindämmung von Falschnachrichten 


\title{
»Fake News«: neue Bedrohung oder alter Hut? Grundlagen für ein Strategisches Diskursmanagement
}

\author{
Julian Hajduk \& Natascha Zowislo-Grünewald
}

Abstract

Der Artikel analysiert - aufbauend auf den Herausforderungen durch Social Media und »Fake News" - disziplinübergreifend die bekannten Kriterien zur Messung von Glaubwürdigkeit. Sodann ergänzt er diese Dimensionen um ein neues Konzept, welches Glaubwürdigkeit von Glauben unterscheidet und plädiert dafür, dass die kommunikationswissenschaftliche Analyse nur unter Berücksichtigung dieser Differenz praktisch umsetzbare und handlungsrelevante Ergebnisse liefert.

\section{Das Problem mit dem Problem}

Mr. Chairman and members of the committee, my name is Nayirah, and I just came out of Kuwait. While I was there, I saw the Iraqi soldiers come into the hospital with guns. They took the babies out of the incubators, took the incubators, and left the children to die on the cold floor. [crying] It was horrifying. (Walton, 1995, 771)

Nach dieser Aussage der kuwaitischen Hilfskrankenschwester Nayirah am 10. Oktober 1990 vor einem inoffiziellen Menschenrechtskomittee des USKongresses geraten verschiedene Dinge ins Rollen: So erwähnt in den nächsten Wochen der damalige US-Präsident George H. W. Bush den Vorfall mehrmals öffentlich, um auf die für die Weltgemeinschaft untragbare Situation in Kuwait hinzuweisen. Auch nationale und internationale Medien greifen den Skandal auf, verbreiten und diskutieren ihn. Zuletzt nimmt Amnesty International den Vorfall in ihren Bericht über Menschenrechtsverletzungen durch das Hussein-Regime auf (Walton, 1995).

Retrospektiv gilt die Aussage Nayirahs deshalb als ein entscheidender Baustein für die Zustimmung sowohl des amerikanischen Senats als auch des Repräsentantenhauses zur am 17. Januar 1991 beginnenden Militäroperation Desert Storm (Walton, 1995). Wie sich erst nach dem Krieg herausstellte, handelte es sich bei Nayirah allerdings nicht um eine Krankenschwester, sondern um die damals 15-jährige Tochter des kuwaitischen USBotschafters Saud Nasir as-Sabah - und ihre Geschichte war nicht nur frei 
erfunden, sondern von der amerikanischen PR-Agentur Hill \& Knowlton regelrecht inszeniert worden (Walton, 1995).

Viel ist über diese, im Nachgang als Brustkastenlüge bekannt gewordene Episode der Macht der Geschichten, Bilder und Medien veröffentlicht und berichtet worden, insbesondere auf der Metaebene: über die konzertierte Manipulation der Presse durch die US-Regierung nach dem VietnamTrauma oder über die gezielte Konstruktion des Feindbildes Saddam Hussein (MacArthur, 1993; Plambach \& Kempf, 1994).

Aus heutiger Sicht ist deshalb interessant, dass ein im derzeitigen öffentlichen Diskurs sehr prominenter Begriff innerhalb der wissenschaftlichen Aufarbeitung der 1990er-Jahre nicht gebräuchlich war. So wird im Kontext des Golfkriegs von Manipulation gesprochen, von Lüge, von »Funktionalisierung « (Meder, 1994) - aber nicht von »Fake News«.

Laut Duden bezeichnet der Begriff »in den Medien und im Internet, besonders in den sogenannten Social Media, in manipulativer Absicht verbreitete Falschmeldungen« (Dudenredaktion, o.J.), womit die Brutkastenlüge alle Charakteristika besitzt, um als »Fake News« zu gelten. Auch handelt es sich nicht um einen Neologismus des 21. Jahrhunderts; der Begriff »Fake News « ist bereits im 19. Jahrhundert - freilich ohne die Emphase Internet - in seiner obenstehenden Bedeutung bekannt (Tworek, 2019). Es scheint also, als sei es tatsächlich der Spezifikation besonders in den sogenannten Social Media beziehungsweise dem Internet selbst geschuldet, dass in unseren Tagen aus Lügen nunmehr »Fake News« geworden sind.

Im Zuge der wissenschaftlichen Urbarmachung der sich so rasant und plötzlich entwickelnden digitalen Sphäre ist die Gefahr einer Begriffshyperinflation bei gleichzeitiger Definitionsarmut inhärent, wie Sven Engesser (2013) formuliert. Dieses Phänomen könnte deshalb auch als Mechanismus hinter dem Aufstieg des Begriffs »Fake News« wirken. Allerdings besteht ebenso die Möglichkeit, dass jene digitale Sphäre genügend Variablen zu sehr verändert hat, um der Bedeutung dessen, was »Fake News« meint, noch mit dem Begriff der Lüge gerecht werden zu können.

Versucht man nun deshalb, die Spezifikation besonders in den Social Media tatsächlich als Distinktionsmerkmal von Lüge und »Fake News« zu gebrauchen, so stellt sich die Frage, was dieses signifikante Unterscheidungsmerkmal seitens der sozialen Medien - hier offensichtlich verwendet in ihrer Bedeutung als Verbreitungsplattform - darstellt.

Bekannt ist in diesem Zusammenhang erstens der Begriff der Filter Bubble. Während ein Zeitungsleser oder Medienkonsument allein durch die redaktionelle Auswahl mit einem polyvalenten Nachrichtenmix konfrontiert wird, selektiert der für die Nachrichtenauswahl auf sozialen Medien 
zuständige Algorithmus lediglich nach den bereits bekannten Präferenzen des Konsumenten. So wird diesem der Blick über den eigenen Tellerrand erschwert und bei Mangel an aktivem Dagegengoogeln verunmöglicht zwangsläufig glaubt er, was er glauben muss. Weil er schlichtweg keine Alternativen kennt.

Zweitens wird kolportiert, dass Social Bots - organisiert in Bot-Netzwerken - durch eine hohe Posting-Frequenz nicht nur den Verbreitungs-, sondern auch den Rezeptionserfolg von »Fake News« signifikant erhöhen. Letzteres erreichen sie, indem die automatisierten Nachrichtenverteiler vorspiegeln, es handle sich bei den eigenen Kommunikationspartnern um menschliche Nutzer, was wiederum die Glaubwürdigkeit der verbreiteten Inhalte automatisch erhöht (Allcott \& Gentzkow, 2017).

Insgesamt lässt sich damit also sagen, dass wohl - wenn auch innerhalb der aktuellen Debatte nicht expressis verbis genannt - von einer durch die Natur sozialer Medien potenzierten Steigerung der Glaubwürdigkeit gesprochen wird, wenn das Phänomen »Fake News« definitorisch erfasst und in seiner Wirkmächtigkeit insbesondere gegen bereits existente Begrifflichkeiten abgegrenzt werden soll. Aus diesem Anlass soll das Konstrukt der Glaubwürdigkeit im massenmedialen Kontext umrissen werden, um darzustellen, ob und wie sich die Parameter im Rahmen der Social-Media-Kommunikation bezüglich der Glaubwürdigkeit von Kommunikation verändert haben.

\section{Glaubwürdigkeit}

Allgemein können wir sagen: Immer dann, wenn Informationen entscheidungsoder handlungsrelevant werden, die uns nicht aus eigener Wahrnehmung bekannt sind, stellt sich prinzipiell die Frage nach deren Glaubwürdigkeit. (Köhnken, 1990, 1)

Da sich die Frage nach der Glaubwürdigkeit - wie schon in der Einführung beschrieben - prinzipiell stellt, wenn Informationen entscheidungs- oder handlungsrelevant werden, muss im Nachfolgenden geklärt werden, was Glaubwürdigkeit überhaupt ist. Die Forschung ist sich dabei - disziplinübergreifend - darüber einig, dass Glaubwürdigkeit ein Konstrukt darstellt und sich somit nicht direkt, sondern nur mit Hilfe verschiedener Indikatoren beobachten lässt (Nawratil, 1997; Shah, Ravana, Hamid \& Ismail, 2015).

Die Bandbreite dieser Indikatoren variiert je nach Disziplin stark, wobei sich diese jedoch stets um die Bereiche der inhaltlichen beziehungsweise Quellenglaubwürdigkeit gruppieren. Hiermit ist gemeint, dass einerseits der vermittelnden Quelle - dem Sender - eine gewisse Glaub- beziehungsweise 
Unglaubwürdigkeit zugesprochen wird, andererseits aber auch dem vermittelten Inhalt.

\section{Quellenglaubwürdigkeit}

Die Glaubwürdigkeit einer Quelle lässt sich gemeinhin mit dem Begriff des Prestiges beschreiben (Nawratil, 1997). Dabei ist für die Glaubwürdigkeit eines bestimmten Kommunikats entscheidend, ob die Quelle innerhalb der zugehörigen respektive zugewiesenen Peer Group Prestige besitzt, nicht ob sie dies allgemein innehat. So wird einem Akademiker zugetraut, dass dieser sich über wissenschaftliche Themen glaubwürdig äußern kann, nicht jedoch zwangsweise zu einem Fußballspiel (Eisend, 2006; Stone \& Hoyt, 1974). Diese Prestige-Dimension lässt sich damit auch als - zugeschriebene - Kompetenz bezeichnen und verweist auf die inhaltliche Dimension von Quellenglaubwürdigkeit.

Hinsichtlich des persönlichen Prestiges einer Quelle existiert dabei auch ein wohl am besten mit dem Begriff Universalgelehrtenstatus zu umschreibendes Phänomen: Personen (des öffentlichen Lebens), welche (durch die öffentliche Meinung) als besonders gebildet respektive kenntnisreich (wahrgenommene Intelligenz, Autorität, Fähigkeit zu informieren; Infante, Rancer \& Womack, 1993) gelten, können sich glaubwürdiger auch zu solchen Themen äußern, welche nicht mit ihrer genuinen Expertise zusammenhängen - dies gilt beispielsweise auch für Institutionen (wie die sogenannten Qualitätsmedien; Hovland, Janis \& Kelley, 1953).

Gleichzeitig kennt Prestige auch eine quantitative Dimension, welche sich direkt mit den Kriterien der wahrgenommenen Intelligenz, Autorität sowie der Fähigkeit zu informieren koppeln lässt: der aus den Wissenschaften bekannte Impact Factor. So wurde zum Beispiel hinsichtlich der Glaubwürdigkeit von Online-Quellen nachgewiesen, dass die Backlink-Struktur respektive die Häufigkeit der Nennung einer Seite oder Person auf weiteren Seiten ein entscheidendes Glaubwürdigkeitskriterium darstellt (Shah, Ravana, Hamid \& Ismail, 2015).

Zweitens genießt eine Quelle rezipientenseitige Vertrauenswürdigkeit, die sich erst innerhalb und mittels der zeitlichen Dimension entfaltet. Eine (positiv ausgeprägte) Vertrautheit mit jemandem erhöht direkt die Glaubwürdigkeit, welche man den von dieser Person gesendeten Botschaften zuspricht (Nawratil, 1997). Hierbei muss jedoch ergänzt werden, dass nicht allein eine Erfahrung über Zeit Vertrauenswürdigkeit kreiert, sondern dass ergänzende, teilweise empirisch noch nicht gesicherte Faktoren ebenfalls 
zu Vertrauenswürdigkeit beitragen (welche damit wieder eher einem Konstrukt ähneln würde): Zuverlässigkeit, Seriosität, Spontaneität oder die von Aristoteles postulierte Reinheit des Motivs (Nawratil, 1997).

Das Zusammenspiel beider Faktoren ist dabei lediglich unzureichend untersucht. So legt eine Studie nahe, dass Vertrauenswürdigkeit die wichtigere der beiden Dimensionen darstellt (McGinnies \& Ward, 1980), wobei bestimmte Sub-Sets diese Priorisierung wieder aufheben (sogenannte Konvertiten beispielsweise gelten aufgrund ihrer persönlichen Erfahrung als besonders glaubwürdige Experten; Levine \& Valle, 1975).

\section{Inhaltliche Glaubwürdigkeit}

Die Glaubwürdigkeit der Botschaft selbst, die inhaltliche Glaubwürdigkeit, lässt sich erneut durch zwei Dimensionen beschreiben:

So konstituiert sich die Glaubwürdigkeit eines Kommunikats einerseits quantitativ. Dies beschreibt das klassische Forschungsgebiet der Kriminalistik, wobei Zeugenaussagen glaubwürdiger (und auch häufiger wahr) sind, je mehr - gerade nebensächliche - Details sie enthalten (Arntzen 1993; Bender 1987). Gleichzeitig wird quantitative Glaubwürdigkeit indirekt auch innerhalb journalistischer Arbeitsroutinen durch den W-Fragen-Standard sichtbar (der auch außerhalb der journalistischen Sphäre als hilfreiches Tool zur vollständigen Detektion inhaltlicher Information dient): Wer? Was? Wo? Wann? Wie? Warum? Woher (ist die Information)? (Schneider \& Raue 2009; Hooffacker 2010; Cappon 2005).

Andererseits wird die Glaubwürdigkeit eines Kommunikats auch durch eine qualitative Dimension bestimmt, welche sich jedoch - im Gegensatz zum positiv korrelierten Verhältnis von Glaubwürdigkeit und Quantität nicht derart einfach fassen lässt. Stattdessen vereinen sich an dieser Stelle viele - teils widersprüchliche - Eigenschaften:

Analog zur Quellenglaubwürdigkeit kennt auch die inhaltliche Glaubwürdigkeit das Kriterium der Vertrauenswürdigkeit, welche sich ebenfalls innerhalb und mittels der zeitlichen Dimension entfaltet. Basal bedeutet dies, dass der Inhalt eines Kommunikats stimmig respektive homogen sein sollte. ${ }^{1}$ Diese Homogenität wird zuallererst durch eine gewisse Widerspruchsfreiheit erzeugt, welche wiederum in der Kriminalistik untersucht wird und mit der Einhaltung grundsätzlicher Kriterien der Aussagen- und

1 Dies gilt für ein einzelnes wie auch mehrere inhaltlich zusammenhängende Kommunikate. 
Argumentationslogik bezeichnet werden könnte (Arntzen, 1993; Bender, 1987).

Zusätzlich existieren noch zahlreiche weitere Kriterien, welche - empirisch bisher nur indirekt nachgewiesen - Homogenität konstituieren und dabei durchaus mit aussagenlogischen Bedingungen brechen können. Diese Kriterien wurden einerseits innerhalb kognitionspsychologischer Studien als sogenannte Memorabilitätsstützen nachgewiesen, andererseits innerhalb der Medien- und Kommunikationswissenschaften im Rahmen der Medienwirkungsforschung als entscheidend für Agenda Setting, Priming und Framing wahrgenommen (Schenk, 2007, Matthes, 2007).

Ähnlichkeit, die Einordnung eines neuen Sachverhalts in eine passende kognitive Schublade, legt einen Grundstein für das individuelle Empfinden von Homogenität und wirkt so vertrauensbildend. Da das menschliche Gedächtnis schematisch angelegt zu sein scheint, kann und wird es sich Dinge, welche es einzuordnen in der Lage ist, eher und besser merken als solchen Input, der kategorisch neu ist (Fiske \& Taylor 1991; Scheufele, 2003).

Eine Vertiefung des Kriteriums Widerspruchsfreiheit stellt die Attributionstheorie in Form der Ursächlichkeit bereit. Ebenfalls durch die Kognitionspsychologie nachgewiesen ist der Umstand, dass sich das menschliche Gedächtnis diejenigen Sachverhalte eher und besser merkt, welche klare Wirkzusammenhänge aufweisen (warum - darum). Dies gilt insbesondere, wenn die Wirkzusammenhänge personal statt situativ sind (jemand statt etwas ist verantwortlich; Jecker 2014; Kelley 1973).

Lediglich erwähnt bleiben an dieser Stelle der Einfluss stilistischer Elemente aus der Erzählforschung und Narratologie: Was eine gute Geschichte ausmacht, die von ihren Lesern geglaubt wird, weil sie bestimmten inhaltlichen wie formellen Stilregeln folgt, ist dabei zwar hinreichend bekannt, nicht jedoch experimentell untersucht hinsichtlich der Glaubwürdigkeit von (Fake) News beziehungsweise sogenannter Tatsachenberichte. ${ }^{2}$

Wenn aus Glaubwürdigkeit Glaube wird: Minimal-Group und Diskursumwelten

Glaubwürdigkeit speist sich somit einerseits aus der Quelle, andererseits aus der Botschaft selbst. Dabei lassen sich qualitative sowie quantitative Ausprägungen der Dimensionen Prestige und Vertrauenswürdigkeit identifizieren. Gleichzeitig ist damit aber noch nicht geklärt, noch nicht einmal 
untersucht, warum der Empfänger einer Botschaft und ihrer Quelle glaubt - oder auch nicht glaubt. Denn unzweifelhaft können all die oben genannten Kriterien gleichzeitig auf beides zutreffen - auf »True News« oder »Fake News«, wenn man diese indes überhaupt objektiv trennscharf klassifizieren kann.

So wird durch die Listung und Differenzierung der Glaubwürdigkeitskriterien klar, dass es mithilfe des Internets beziehungsweise der sozialen Medien wesentlich leichter ist als noch im Zeitalter der klassischen Massenmedien, Wahrheit zu manipulieren. Man benötigt zur Schaffung einer gefälschten Backlink-Struktur lediglich einige Programmierkenntnisse - beispielsweise indem man Expertise und damit Glaubwürdigkeit mithilfe eines Twitter-Bots erzeugt (Edwards, Edwards, Spence \& Shelton, 2014). Dies dürfte auch einer der Hauptgründe sein, weshalb das Problem als neuartig empfunden wird - und Antworten darauf umso drängender gesucht werden. Die zugrundeliegenden Wirkmechanismen sind allerdings die gleichen geblieben, wie sie die Soziologie bereits für das Zeitalter der Massenmedien definiert hat:

Andererseits wissen wir so viel über die Massenmedien, dass wir diesen Quellen nicht trauen können. Wir wehren uns mit einem Manipulationsverdacht, der aber nicht zu nennenswerten Konsequenzen führt, da das den Massenmedien entnommene Wissen sich wie von selbst zu einem selbstverstärkenden Gefüge zusammenschließt. Man wird alles Wissen mit dem Vorzeichen des Bezweifelbaren versehen - und trotzdem darauf aufbauen, daran anschließen zu müssen. (Luhmann 1996, 910)

Und so ist zuletzt, wie Nawratil feststellt - und wie es dem konstruktivistischen Paradigma entspricht -, Glaubwürdigkeit »ein zuschreibungsbasiertes Konzept « (Nawratil, 1997, 18). Um den Impact hinter dem Prinzip »Fake News« letztendlich zu begreifen, spielt es deshalb keine Rolle, ob eine Botschaft oder ihre Quelle glaubwürdig sind. Auf diese Weise lässt sich zwar untersuchen, welchen Inhalten wahrscheinlicher geglaubt wird als anderen - und hier sollten im Sinne eines ganzheitlich-strategischen Ansatzes auch mögliche Potenziale ausgeschöpft werden. Gleichzeitig ist der Themenkomplex an sich aber wohl derart (über)komplex, dass die Vielzahl unbekannter Faktoren es (bisher) verunmöglicht, eine Lüge dezidiert zu erkennen - und selbst wenn man dies vermöchte, wäre eine vorhandene Glaubhaftigkeit im Sinne des nach Lüge forschenden Betrachters noch kein Indikator für ihre Wirkmächtigkeit bei ihrer Zielgruppe.

Zwei bekannte - und doch im vorliegenden Kontext - neue Dimensionen sollen daher Klarheit schaffen, um zukünftig den Impact-Faktor von »Fake News« messen zu können. Dies ist einerseits die von Henri Tajfel entwickelte und empirisch im Rahmen der sogenannten Minimal-Group- 
Experimente erfolgreich getestete Theorie der sozialen Identität (Tajfel 1982). Diese überprüft (und bestätigt in gewisser Weise) das post-kantianische Paradigma einer Identität aus Differenz (Clam, 2002).

Was von der Systemtheorie mithilfe des Imperativs »Triff eine Entscheidung!« zusammengefasst wird, überträgt das Minimal-Group-Experiment auf die soziale Sphäre: So tendieren Individuen dazu, selbst im Angesicht völlig trivialer Unterscheidungsmerkmale (rot-Team und gold-Team, sogenannte Minimal Groups) Mitglieder der eigenen Gruppe zu bevorzugen, wenn es beispielsweise um die Verteilung von Geldgeschenken oder die Bewertung von Persönlichkeitsmerkmalen geht. Dieser Effekt ist auch dann zu beobachten, wenn Probanden den Gruppen vollkommen zufällig zugeteilt werden und diese ansonsten keinerlei Kenntnis über die jeweils anderen Teilnehmer des eigenen und fremden Teams besitzen (Tajfel, Billig, Bundy \& Flament, 1971; Billig \& Tajfel, 1973).

Individuen ordnen sich also $\mathrm{zu}$ - bevor sie irgendetwas anderes tun. Folgt man nun dem - wie bereits erwähnt - zugrundeliegenden Metakonzept des sozialen Konstruktivismus, so müssen sie dies auch. Einerseits besteht hierfür eine epistemologische Notwendigkeit (ich bin - jedoch nur in Abgrenzung zu jemand anderem), andererseits knüpft diese Identifikation - NichtIdentifikation auch an die Theorie des sozialen Kapitals an. Zugehörigkeit schafft also nicht nur Identifikation (und vice versa), sondern - über das Mittel der Gruppenzugehörigkeit - auch soziales Prestige. Dieses von James Coleman als Makro-Mikro-Makro-Wirkschema beschriebene Phänomen kann im Zusammenhang mit den Erkenntnissen aus der Theorie der sozialen Identität erklären, wie die vorgenannte Eigengruppenfavorisierung im sozialen Kontext funktioniert - und welche Implikationen dies für den Untersuchungsgegenstand »Fake News« zulässt (Coleman, 1990; Hajduk, 2018).

So existieren Glaubenssysteme innerhalb der eigenen Gruppe, über die sich diese nachhaltig mittels Zuschreibung konstituiert. Diese Diskursumwelten dessen, »was gedacht werden kann und darf«, legen den grundsätzlichen Handlungsrahmen von Akteuren fest. Als Entitäten einer sozialen moyen durée bestimmen sie in groben Zügen die Extrempunkte der politischen, wirtschaftlichen, kulturellen und religiösen Agenda einer (Supra-) Gesellschaft, wie schon Tajfel anmerkt:

Der Erwerb der Vorstellung von einem Wertunterschied zwischen der eigenen Gruppe (oder den eigenen Gruppen) und anderen Gruppen ist ein integraler Bestandteil des allgemeinen Sozialisierungsprozesses. [...] Aus diesem Blickwinkel betrachtet kann die soziale Kategorisierung somit als ein Orientierungssystem angesehen werden, das dazu beiträgt, den Platz des Individuums in der Gesellschaft zu schaffen und zu definieren [...]. (Tajfel, 1982, 102-103) 
Diese als im weitesten systemtheoretischen Sinne binär zu interpretierenden Standpunkte erlauben Zuschreibung und Zuordnung - häufig verbunden mit einem ex negativo-Gegenpol: Man ist politisch für Donald Trump (und seine Politik) oder gegen ihn (und seine Politik). Man ist ein Klimaaktivist - oder ein Leugner. Man ist gläubig/religiös - oder aufgeklärt/agnostisch. Das klar umrissene Contra ist dabei nicht einmal notwendig; was das Für und Wider eines Standpunkts ausmacht, ist - impressis verbis - Allgemeinwissen (»Wer den Klimawandel leugnet, wählt auch Donald Trump « beziehungsweise »Wenn du Donald Trump wählst, lehnst du sicherlich auch die gleichgeschlechtliche Ehe ab«). Der Diskurs innerhalb der Berichterstattung zur europäischen Banken- und Staatsfinanzkrise changierte beispielsweise zwischen den Extremen von Interventionismus und (nationalstaatlichem) Protektionismus, während sozialistische oder wirtschaftsliberale Standpunkte kaum eine Rolle spielten (Hajduk 2018).

Somit richtet sich Glaubwürdigkeit als zuschreibungsbasiertes Konzept zuallererst nach diesen basal vorgenommenen Kategorisierungen aus. Zwar erschaffen die Medien die Welt, in der wir leben. Gleichzeitig darf dabei aber keinesfalls vergessen werden, dass auch die Medien nur jene Welt erschaffen, von der sie sich erhoffen, dass wir darin leben und von welcher wir erfahren wollen (Luhmann, 1996). Deshalb ist es wichtig, »Fake News « nicht nur zu erkennen, sondern auch den fruchtbaren (oder fruchtlosen) Boden zu untersuchen, auf dem sie gedeihen (können).

\section{Strategisches Diskursmanagement als holistisches Konzept}

Hans Vaihinger beschreibt in seiner Philosophie des »Als Ob«Fiktionen als Konstruktionen einer unwirklichen Wirklichkeit in allen möglichen Bereichen des menschlichen Lebens. Auf vielen Gebieten handelten die Menschen, als ob ihre Fiktionen wahr wären. Rein pragmatisch gesprochen spielt für das Handeln der Menschen die Sphäre objektiver Wahrheit somit keine Rolle. An die Stelle des absoluten Wahrheitsbegriffs treten zweckmäßige Fiktionen. Sie geben den Menschen Orientierung im Handeln, oder wie es William Isaac Thomas ausdrückt: »If men define situations as real, they are real in their consequences « - und vice versa (Thomas \& Thomas, 1928, 572; Vaihinger, 1911).

Das Beispiel der Brutkastenlüge beschreibt somit nichts anderes als eine geglückte, nach den Regeln der Kunst durchgeplante und exerzierte Kampagne - deren Wirkung schlussendlich aber nur entfaltet werden konnte, da sie - zumindest von großen Teilen der meinungsbildenden Diskurselite 
einerseits sowie großen Teilen der meinungsbildenden Mehrheit andererseits geglaubt werden wollte. Und es darf weiterhin gemutmaßt werden, dass auch dies der Grund ist, warum die Entdeckung der Lüge, der »Fake News«, sodann nicht mehr von grundsätzlichem öffentlichen Interesse war und sich an der Darstellung der Ereignisse nichts Grundlegendes verändert hat: der Frame, die Rahmung der Ereignisse, hat klare, stringente und argumentationslogische Ursachen und Wirkungen zugewiesen, ein klar akzeptiertes Gut und Böse verteilt - und somit eine stabile interpretatorische Struktur geschaffen, welche sogar einer Lüge widersteht und damit Phänomene wie einen später eintretenden Hostile Media Effect erklären kann (Vallone, Ross \& Lepper, 1985).

Entscheidend für erfolgreiches Kommunikationsmanagement ist damit tatsächlich nicht die Frage nach der Wahrheit. Im Gegenteil: Die Wahrheit erschüttert im Zweifelsfall sogar bereits bestehende vertrauensvolle Beziehungen, wenn sie nicht anschlussfähig ist und existierende Rahmungen zu brechen versucht - oder aber, sie wird an der Stabilität dieser Rahmungen sogar schlichtweg scheitern. Deshalb gilt es zu betonen, dass sich die Wahrheit - so man denn von ihr sprechen möchte - an der Welt, dem Diskursangebot ihrer verschiedenen Stakeholder orientiert, da Realität keine den Dingen anhaftende intrinsische Qualität darstellt, sondern entscheidend von glaubhaften Interpretationen abhängt.

Weiterhin lässt sich die Metapher der produktiven Fiktion ökonomisch fassen - genauso wie Foucaults und Tajfels Theorien des sozialen Kapitals respektive der sozialen Identität es ja bereits verlangen: Weil Informationsbeschaffung und -bewertung exponentiell sich steigernde Kosten verursachen, welche schon sehr bald in Überkomplexität münden, ist die Folgsamkeit gegenüber der Irrationalität für den einzelnen Akteur rationaler, als im Sinne des Problems der doppelten Kontingenz - überhaupt keine Entscheidung mehr treffen zu können (Luhmann, 1989; Downs, 1957).

Insofern muss der Ansatz zukünftiger Untersuchungsschemata weg von der Differenz Wahrheit/Lüge hin zum Begriffspaar glaubwürdig/unglaubwürdig (für eine homogene Stakeholder-Gruppe) verschoben werden. So wird einerseits dem Sender-Botschaft-Gefüge inhärenten Faktorenbündel zur Detektion von Glaubwürdigkeit Rechnung getragen, andererseits die konstituierende Rolle der Lebenswelt der gesellschaftlichen (Gegen-)Gruppierungen und der aus ihnen hervorkeimenden themen- und gruppenspezifischen Diskurseinheiten von dem, was vermeintlich geglaubt und gesagt werden darf notwendigerweise mit in die Untersuchung einbezogen: »There is a sense in which what is play for the golfer is work for the caddy « Goffman, 1974, 8). 
Insbesondere in den Zeiten von Social Media, wo das ungleich größere und leichter zugängliche Angebot sowie ebenjene potenzierte Wichtigkeit der Kriterien von inhaltlicher und Quellenglaubwürdigkeit so deutlich Zünglein an der Waage im Kampf um Deutungshoheit darstellen, wird durch das Festhalten an absoluten Wahrheitsbegriffen deshalb auch nur ebenjener Effekt ausgelöst, welcher sogenannten gesättigten Frames inhärent ist: das unbedingte Festhalten an der stabilen kognitiven Ingroup und die Ablehnung anderer (noch so überzeugender) Outgroups. Bildet der Glaube - analog zur Glaubwürdigkeit - einen Faktor, welcher von den Dimensionen Minimal-Group und Diskurs getragen wird, so leitet sich daraus auch deren Komplementarität ab.

Insofern wäre im Rahmen von Handlungsempfehlungen ein erster Schritt zum Überschreiten der Grenze zu den Anderen auch nicht, deren tatsächliche oder vermeintliche Lügen als solche zu entlarven, sondern - wenig invasiv - den eigenen Diskurs, die vermeintliche Wahrheit, zu übersetzen in die Sprache derjenigen, die ihn bisher nicht verstehen - respektive nicht einmal denken - können.

\section{Literatur}

Allcott, Hunt \& Gentzkow, Matthew (2017). Social Media and Fake News in the 2016 Election. Journal of Economic Perspectives, 31(2), 211-236. DOI: 10.1257/jep.31.2.211.

Arntzen, Friedrich (1993). Psychologie der Zeugenaussage. System der Glaubwürdigkeitsmerkmale. München: C.H. Beck.

Bender, Hans-Udo (1987). Merkmalskombinationen in Aussagen. Theorie und Empirie zum Beweiswert beim Zusammentreffen von Glaubwürdigkeitskriterien. Tübingen: Mohr Siebeck.

Billig, Michael \& Tajfel, Henri (1973). Social categorization and similarity in intergroup behavior. European Journal of Social Psychology, 3(1), 27-52. DOI: 10.1002/ejsp.2420030103.

Campbell, Joseph (1949). The Hero with a Thousand Faces. New York: Pantheon Books.

Cappon, Rene (2005). Associated Press Handbuch. Journalistisches Schreiben. Berlin: Autorenhaus.

Chatman, Seymour (1978). Story and Discourse. Narrative Structure in Fiction and Film. Ithaca/London: Cornell University Press.

Clam, Jean (2002). Was heißt, sich an Differenz statt an Identität zu orientieren? Zur De-ontologisierung in Philosophie und Sozialwissenschaft. Konstanz: UVK.

Coleman, James (1990). Foundations of Social Theory. Cambridge: Belknap Press. 
Downs, Anthony (1957). An Economic Theory of Political Action in Democracy. Journal of Political Economy 65(2), 135-150. DOI: 10.1086/257897.

Dudenredaktion (o. A.). Fake News auf Duden Online. Abgerufen von https://www. duden.de/node/44637/revision/44666. Abgerufen am 04. Februar 2020.

Edwards, Chad; Edwards, Autumn; Spence, Patric \& Shelton, Ashleigh (2014). Is That a Bot Running the Social Media Feed? Testing the Differences in Perceptions of Communication Quality for a Human Agent and a Bot Agent on Twitter. Computers in Human Behavior 33, 372-376. DOI: 10.1016/j.chb.2013.08.013.

Eisend, Martin (2006). Source Credibility Dimensions in Marketing Communication A Generalized Solution. Journal of Empirical Generalisations in Marketing Science, 10(2), 1-33.

Engesser, Sven (2013). Die Qualität des Partizipativen Journalismus im Web. Bausteine für ein integratives theoretisches Konzept und eine explanative empirische Analyse. Wiesbaden: Springer VS.

Fiske, Susan \& Taylor, Shelley (1991). Social Cognition. New York: McGraw-Hill.

Freytag, Gustav (1863). Die Technik des Dramas. Leipzig: S. Hirzel.

Goffman, Erving (1974). Frame Analysis. Boston: Northeastern University Press.

Hajduk, Julian (2018). Vom Bewusstsein des Möglichen. Eine Framing-Analyse zur Darstellung der Banken- und Staatsfinanzkrise in deutschen Online-Medien. Abgerufen von https://opus4.kobv.de/opus4-udk/frontdoor/deliver/index/docId/1211/file/ Das_Bewusstsein_des_Moeglichen_gutachter.pdf. Abgerufen am 18. September 2019 .

Hooffacker, Gabriele (2010). Online-Journalismus. Texten und Konzipieren für das Internet. Ein Handbuch für Ausbildung und Praxis. Berlin: Econ.

Hovland, Carl; Janis, Irving \& Kelley, Harold (1953). Communication and Persuasion. Psychological Studies of Opinion Change. New Haven: Yale University Press.

Infante, Dominic; Rancer, Andrew \& Womack, Deanna (1993). Building Communication Theory. Illinois: Waveland Press.

Jecker, Constanze (2014). Entmans Framing-Ansatz. Theoretische Grundlegung und empirische Umsetzung. Konstanz: UVK.

Kelley, Harold (1973). The Processes of Causal Attribution. American Psychologist, 28(2), 107-128. DOI: 10.1037/h0034225.

Köhnken, Günter (1990). Glaubwürdigkeit. Untersuchungen zu einem psychologischen Konstrukt. München: Psychologie Verlags Union.

Levine, John \& Valle, Ronald (1975). The Convert as a Credible Communicator. Social Behaviour and Personality, 3(1), 81-90. DOI: 10.2224/sbp.1975.3.1.81.

Luhmann, Niklas (1989). Vertrauen. Ein Mechanismus der Reduktion sozialer Komplexität. Stuttgart: Enke.

Luhmann, Niklas (1996). Die Realität der Massenmedien. Opladen: Westdeutscher Verlag.

Matthes, Jörg (2007). Framing-Effekte. Zum Einfluss der Politikberichterstattung auf die Einstellung der Rezipienten. München: Reinhard Fischer. 
McGinnies, Elliott \& Ward, Charles (1980). Better Liked Than Right: Trustworthiness and Expertise as Factors in Credibility. Personality and Social Psychology Bulletin, 6(3), 467-472. DOI: 10.1177/014616728063023.

MacArthur, John (1993). Die Schlacht der Lügen. Wie die USA den Golfkrieg verkauften. München: dtv.

Meder, Gerhard (1994). Die Funktionalisierung der Vereinten Nationen. In: Kempf, W. (Hrsg.), Manipulierte Wirklichkeiten. Medienpsychologische Untersuchungen der bundesdeutschen Presseberichterstattung im Golfkrieg (139-157). Münster \& Hamburg: LIT.

Nawratil, Ute (1997). Glaubwürdigkeit in der Sozialen Kommunikation. Opladen: Westdeutscher Verlag.

Palmbach, Ute \& Kempf, Wilhelm (1994). Die Konstruktion des Feindbildes Saddam. In: Kempf, W. (Hrsg.), Manipulierte Wirklichkeiten. Medienpsychologische Untersuchungen der bundesdeutschen Presseberichterstattung im Golfkrieg (58-81). Münster/Hamburg: LIT.

Propp, Wladimir (1972). Die Morphologie des Märchens. München: Hanser.

Schenk, Michael (2007). Medienwirkungsforschung. Tübingen: Mohr-Siebeck.

Scheufele, Bertram (2003). Frames - Framing - Framing-Effekte. Theoretische und methodische Grundlegung des Framing-Ansatzes sowie empirische Befunde zur Nachrichtenproduktion. Wiesbaden: Westdeutscher Verlag.

Schneider, Wolf \& Raue, Paul-Josef (2009). Das neue Handbuch des Journalismus. Reinbek: Rowohlt.

Shah, Asad; Ravana, Sri Devi; Hamid, Suraya \& Ismail, Maizatul (2015). Web Credibility Assessment: Affecting Factors and Assessment Techniques. Information Research, 20(1), Paper 655. Abgerufen von http://InformationR.net/ir/20-1/paper655.html. Abgerufen am 16. September 2019.

Stein, Sol (1995). Stein on Writing. New York: St. Martin's Press.

Stone, Vernon \& Hoyt, James (1974). The Emergence of Source-Message Orientation as a Communication Variable. Communication Research, 1(1), 89-109. DOI: $10.1177 / 009365027400100105$.

Tajfel, Henri; Billig, Michael; Bundy, R.P. \& Flament, Claude (1971). Social Categorization and Intergroup Behavior. European Journal of Social Psychology, 1(2), 149178. DOI: $10.1002 /$ ejsp.2420010202.

Tajfel, Henri (1982). Gruppenkonflikt und Vorurteil. Entstehung und Funktion sozialer Stereotypen. Bern, Stuttgart, Wien: Hans Huber.

Thomas, William Isaac \& Thomas, Dorothy Swaine (1928). The child in America: Behavior problems and programs. New York: Knopf.

Tworek, Heidi (2019). News from Germany. The Competition to Control World Communications, 1900-1945. Cambridge (Massachusetts)/London: Harvard University Press.

Vahinger, Hans (1911). Die Philosophie des Als Ob. System der theoretischen, praktischen und religiösen Fiktionen der Menschheit auf Grund eines idealistischen Positivismus; mit einem Anhang über Kant und Nietzsche. Berlin: Reuther \& Reichard. 
Vallone, Robert; Ross, Lee \& Lepper, Mark 1985). The Hostile Media Phenomenon. Biased Perception and Perceptions of Media Bias in Coverage of the Beirut Massacre. Journal of Personality and Social Psychology, 49(3), 577-585. DOI: 10.1037//00223514.49.3.577.

Walton, Douglas (1995). Appeal to Pity. A Case Study of the Argumentum Ad Misericordiam. Argumentation, 9, 769-784. DOI: 10.1007/BF00744757. 